\title{
Classical Conditioning Analog Enhanced Acetylcholine Responses But Reduced Excitability of an Identified Neuron
}

\author{
Fred D. Lorenzetti, Douglas A. Baxter, and John H. Byrne \\ Department of Neurobiology and Anatomy, W.M. Keck Center for Neurobiology of Learning and Memory, The University of Texas Medical School at \\ Houston, Houston, Texas 77030
}

\begin{abstract}
Although classical and operant conditioning are operationally distinct, it is unclear whether these two forms of learning are mechanistically distinct or similar. Feeding behavior of Aplysia provides a useful model system for addressing this issue. Both classical and operant appetitive behavioral training enhance feeding, and neuronal correlates have been identified. Behavioral training was replicated by in vitro analogs that use isolated ganglia. Moreover, a single-cell analog of operant conditioning was developed using neuron B51, a cell important for the expression of the conditioned behavior. Here, a single-cell analog of classical conditioning was developed. Acetylcholine (ACh) mediated the conditioned stimulus (CS)-elicited excitation of B51 in ganglia and mimicked the CS in the single-cell analog of classical conditioning. Pairing ACh with dopamine, which mediates the unconditioned stimulus in ganglia, decreased the excitability of $\mathrm{B} 51$, and increased the CS-elicited excitation of B51, similar to results following both in vivo and in vitro classical training. Finally, a $\mathrm{D}_{1}$ dopamine receptor $\left(D_{1} R\right)$ agonist failed to support classical conditioning in the cellular analog, whereas $D_{1} R$ mediates reinforcement in operant conditioning.
\end{abstract}

\section{Introduction}

Classical conditioning involves the ability to associate a predictive stimulus [conditioned stimulus (CS)] with a subsequent salient event [unconditioned stimulus (US)], whereas operant conditioning involves the ability to associate an expressed behavior with its consequences. These two forms of associative learning allow for a predictive understanding of a changing environment. Although they are operationally distinct, it is unclear whether classical and operant conditioning are mechanistically distinct or similar (Rescorla and Solomon, 1967).

The feeding behavior of Aplysia provides a useful model system for comparative analyses of the mechanisms underlying classical and operant conditioning (Baxter and Byrne, 2006; Nargeot and Simmers, 2011). Feeding can be increased by appetitive forms of operant and classical conditioning in vivo (Lechner et al., 2000; Brembs et al., 2002; Lorenzetti et al., 2006; Nargeot et al., 2007), and fictive feeding can be increased by in vitro analogs of conditioning (Nargeot et al., 1997, 1999b,c; Mozzachiodi et al., 2003, 2008; Reyes et al., 2005). Moreover, correlates of both forms of learning were characterized in neuron B51 (Brembs et al., 2002; Lorenzetti et al., 2006; Mozzachiodi et al., 2008), a cell

\footnotetext{
Received March 11, 2011; revised Aug. 4, 2011; accepted Aug. 22, 2011.

Author contributions: F.D.L., D.A.B., and J.H.B. designed research; F.D.L., D.A.B., and J.H.B. performed research;

F.D.L., D.A.B., and J.H.B. analyzed data; F.D.L., D.A.B., and J.H.B. wrote the paper.

This work was supported by National Institute of Mental Health Grant R01-MH58321.

The authors declare no competing financial interests.

Correspondence should be addressed to John H. Byrne, Department of Neurobiology and Anatomy, W.M. Keck

Center for the Neurobiology of Learning and Memory, The University of Texas Medical School at Houston, 6431 Fannin Street, MSB 7.046, Houston, TX 77030. E-mail: John.H.Byrne@uth.tmc.edu.

F. D. Lorenzetti's present address: Cellular Neurobiology Research Branch, Intramural Research Program, National Institute on Drug Abuse, 251 Bayview Boulevard, Baltimore, MD 21224

DOI:10.1523/JNEUROSCI.1256-11.2011

Copyright $\odot 2011$ the authors $\quad 0270-6474 / 11 / 3114789-05 \$ 15.00 / 0$
}

that plays a role in the expression of fictive feeding (Nargeot et al., 1999a; Jing et al., 2004; Shetreat-Klein and Cropper, 2004). B51 exhibits an all-or-nothing sustained level of activity (i.e., plateau potential). Operant conditioning alters the intrinsic excitability of B51 by decreasing the threshold for eliciting a plateau potential and increasing the input resistance of the cell. In contrast, classical conditioning increases the threshold for eliciting a plateau potential, while having no effect on input resistance. In addition, classical conditioning increases the CS-induced excitation of B51. These results suggest that operant and classical conditioning operate through distinct cellular and molecular mechanisms. The present study extended the comparative analyses by developing a single-cell analog of classical conditioning to match the previously developed single-cell analog of operant conditioning.

\section{Materials and Methods}

Aplysia californica (80-160 g) specimens were obtained from Alacrity Marine Biological Specimens and Marinus Scientific. Aplysia are hermaphroditic. Animals were housed in perforated plastic cages floating in aerated seawater tanks at a temperature of $15^{\circ} \mathrm{C}$. Animals were fed $\sim 1 \mathrm{~g}$ of dried seaweed three times per week.

Characterization of the CS response in B51. Animals were anesthetized by injecting a volume of isotonic $\mathrm{MgCl}_{2}$ equivalent to $50 \%$ of the weight of the animal. Buccal and cerebral ganglia were removed and pinned on a Sylgard-coated Petri dish containing high-divalent artificial seawater (ASW) to decrease neural activity during dissection. The composition of the high-divalent ASW was as follows (in mM): $\mathrm{NaCl} 210, \mathrm{KCl} 10, \mathrm{MgCl}_{2}$ 145, $\mathrm{MgSO}_{4} 20, \mathrm{CaCl}_{2} 33$, and HEPES 10, $\mathrm{pH}$ adjusted to 7.4 with $\mathrm{NaOH}$.

The most medial and ventral branch of the right anterior tentacle nerve (AT4) was retained, as it innervates the lip region that received the CS during behavioral testing and training (Lechner et al., 2000; Lorenzetti et al., 2006). Electrical stimulation of AT4 was used to mimic the mechanical stimulation of the lips, which represented the CS in a previously developed in vitro analog of classical conditioning (Mozzachiodi et al., 2003; Reyes et al., 2005; Loren- 
zetti et al., 2006). For stimulation, bipolar electrodes were placed on AT4 and isolated from the bath with Vaseline.

The right buccal hemiganglion was desheathed on the rostral side to access the soma of neuron B51. After desheathing, the high-divalent ASW was exchanged for normal ASW, which was composed of the following (in mM): $\mathrm{NaCl} 450, \mathrm{KCl} 10, \mathrm{MgCl}_{2} 30, \mathrm{MgSO}_{4} 20, \mathrm{CaCl}_{2} 10$, and HEPES 10, $\mathrm{pH}$ adjusted to 7.4 with $\mathrm{NaOH}$. The temperature of the bath was maintained at $15^{\circ} \mathrm{C}$. Conventional current-clamp techniques were used for intracellular recordings. Fine-tipped glass microelectrodes (resistance $10-15 \mathrm{M} \Omega$ ) were filled with $2 \mathrm{~m}$ potassium acetate. The soma of neuron B51 was identified by its relative size and position and by its characteristic plateau potential.

Beginning 5 min after impalement, the magnitude of the CS-evoked synaptic input to B51 was measured. The magnitude of the CS-evoked synaptic input to B51 was quantified by delivering an $8 \mathrm{~s}$ duration train ( 5 $\mathrm{Hz}, 0.5$ ms pulses, $10 \mathrm{~V}$ ) to AT4 while the membrane potential of B51 was current-clamped at $-60 \mathrm{mV}$. If a spontaneous motor pattern occurred while recording the synaptic properties of B51, measurements were halted and then resumed $60 \mathrm{~s}$ after the cessation of the pattern. As a control, another identical stimulus was delivered to AT4 10 min later. Next, the acetylcholine (ACh) receptor antagonist hexamethonium (HEX; Sigma) was bath applied to the ganglia at concentrations of either 10 or $100 \mu \mathrm{M}$. The AT4 nerve was stimulated again $10 \mathrm{~min}$ later to measure the CS response in B51 in the presence of hexamethonium. The drug was then washed out with normal ASW, and the AT4 nerve was stimulated again $10 \mathrm{~min}$ later. Cells that initially exhibited CS-evoked EPSPs of $<0.5 \mathrm{mV}$ were discarded.

Single-cell analog of classical conditioning. As previously described, individual B51 cells were maintained in single-cell cultures (Lorenzetti et al., 2008). Conventional techniques were used to current-clamp cultured B51 neurons to $-80 \mathrm{mV}$ for the duration of the experiment. Also, electrodes containing ACh (Sigma) and DA (Sigma) were positioned near the axon hillock region of the B51 neurons. The CS (ACh) and US (DA) were applied to the cell by iontophoresis (see below). Five minutes after impalement, the input resistance, burst threshold, and the initial CS response were determined. Input resistance was measured by injecting a $-0.5 \mathrm{nA}$ current pulse for $5 \mathrm{~s}$. The burst threshold was determined by a series of successively greater amplitude depolarizing current pulses (in $0.1 \mathrm{nA}$ increments) with a duration of $5 \mathrm{~s}$ and with $10 \mathrm{~s}$ between the end of one pulse and the start of another. The response to the CS was measured in B51 by iontophoresing ACh onto the cell for $8 \mathrm{~s}$ (see below). Similar to our previous in vitro analog of classical conditioning (Mozzachiodi et al., 2003; Reyes et al., 2005; Lorenzetti et al., 2006), the protocol for the single-cell analog consisted of 10 pairings of the CS and US. The CS lasted for $8 \mathrm{~s}$, and the US lasted for $4 \mathrm{~s}$. The onset of the US occurred $4 \mathrm{~s}$ after the onset of the CS. The interval between pairings was $2 \mathrm{~min}$ (i.e., an ITI of $2 \mathrm{~min}$ ). The membrane properties and the response to ACh were measured before, immediately after, and $24 \mathrm{~h}$ after training.

DA and ACh were iontophoresed through fine-tipped glass microelectrodes (resistance 10-15 M $\Omega$ ). A retaining current of $-1 \mathrm{nA}$ was used during the course of the experiment. A square wave current pulse of 50 $\mathrm{nA}$ for $8 \mathrm{~s}$ was used to eject the ACh. The concentration of ACh in the electrode was $500 \mathrm{~mm}$. A square wave current pulse of $35 \mathrm{nA}$ for $4 \mathrm{~s}$ was used to eject the DA. The concentration of DA in the electrode was 200 $\mathrm{mM}$. An equimolar concentration of ascorbic acid was added to the electrode to reduce the oxidation of DA. Several lines of evidence indicate that ascorbic acid per se had no detectible effects. First, in a previous study (Kabotyanski et al., 2000), perfusing buccal ganglia with control saline that also contained ascorbic acid had no effect on neural activity. Moreover, in our previous single-cell analog of operant conditioning, noncontingent application of DA solutions with ascorbic acid had no effects on the biophysical properties of B51 (Brembs et al., 2002; Lorenzetti et al., 2008). Finally, iontophoresis of DA in a solution containing ascorbic acid produced a consistent depolarization of the membrane potential, whereas iontophoresis of ascorbic acid alone did not at the concentrations used in this study. In a separate set of experiments, the single-cell analog was also performed by iontophoresing the $\mathrm{D}_{1}$ dopamine receptor $\left(D_{1} R\right)$ agonist chloro-APB (Sigma), instead of DA. The concentration of chloro-APB in the electrode was $100 \mathrm{mM}$.
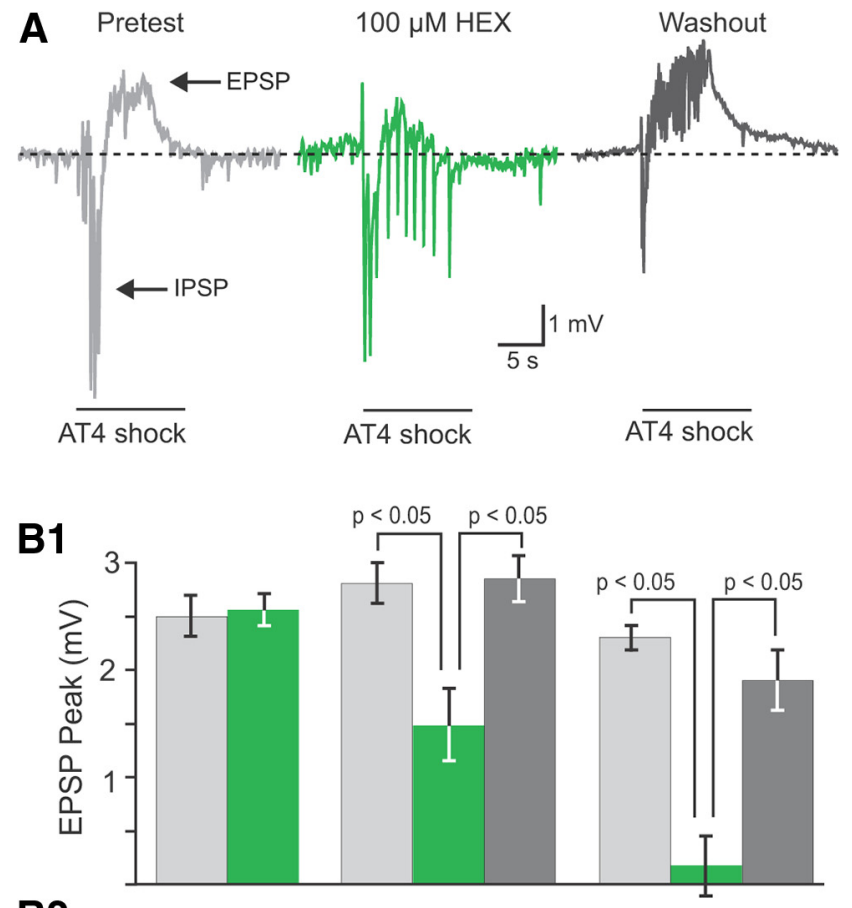

B2

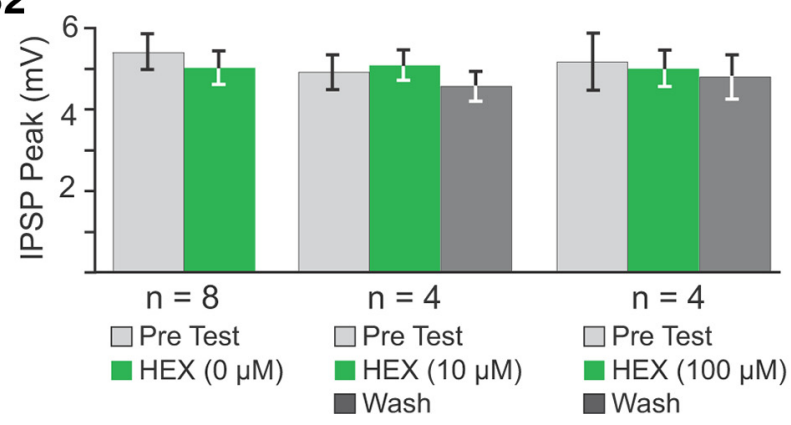

Figure 1. HEX blocked the EPSP in B51 produced by AT4 stimulation in ganglia preparations. The magnitude of the CS-evoked synaptic input to B51 was quantified while B51 was currentclamped at $-60 \mathrm{mV}$. A, Representative intracellular recordings from B51 during AT4 nerve stimulation. AT4 stimulation typically produced a complex PSP in B51 (Pretest) with an initial inhibitory phase followed by a delayed excitatory phase. The EPSP was selectively blocked by HEX (100 $\mu \mathrm{M})$ and recovered following washout (Washout). B, Measurement of the peak IPSP and EPSP at two different doses of hexamethonium. The dashed line represents the baseline potential in B51 (i.e., $-60 \mathrm{mV}$ ) before stimulation of AT4. B1, № significant difference was observed between the peak EPSPs induced by consecutive AT shocks in the absence of HEX ( 0 $\mu \mathrm{M})$. In contrast, the $10 \mu \mathrm{m}$ dose of hexamethonium produced a significant effect $(p<0.05$, Friedman test). A post hoc analysis revealed a significant block of the EPSP ( $p<0.05$, NewmanKeuls test), which recovered ( $p<0.05$, Newman-Keuls test) following the washout. The 100 $\mu \mathrm{m}$ dose of hexamethonium also produced a significant effect ( $p<0.05$, Friedman test). A post hoc analysis revealed a significant block of the $\operatorname{EPSP}(p<0.05$, Newman-Keuls test), which recovered ( $p<0.05$, Newman-Keuls test) following the washout. B2, No significant difference was observed between the peak values of the IPSP at each dose of hexamethonium.

All measurements of membrane properties are displayed as the percentage difference between the post-test and pretest values normalized to the pretest values. All recordings were performed at room temperature $\left(\sim 21^{\circ} \mathrm{C}\right)$

Statistical analysis. All values were expressed as means and SEs. Statistical significance was set at $p<0.05$. Nonparametric statistics were used because we did not have sufficient information on the nature of the population distribution (Siegel, 1956). Comparisons between two dependent samples were made using the Wilcoxon signed-rank test. Comparisons between three dependent samples were made using the Friedman test and post hoc pairwise multiple comparisons were made using the nonparametric Newman-Keuls test. Comparisons between 

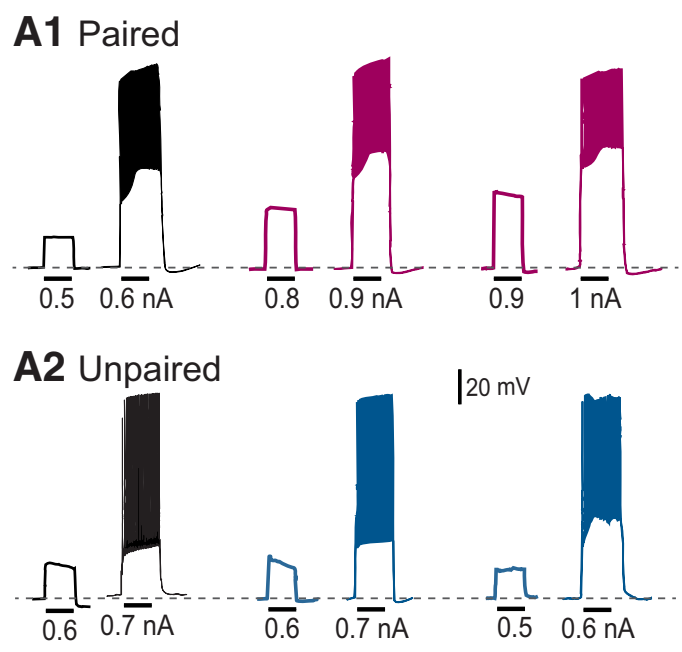

\section{B1 Paired}

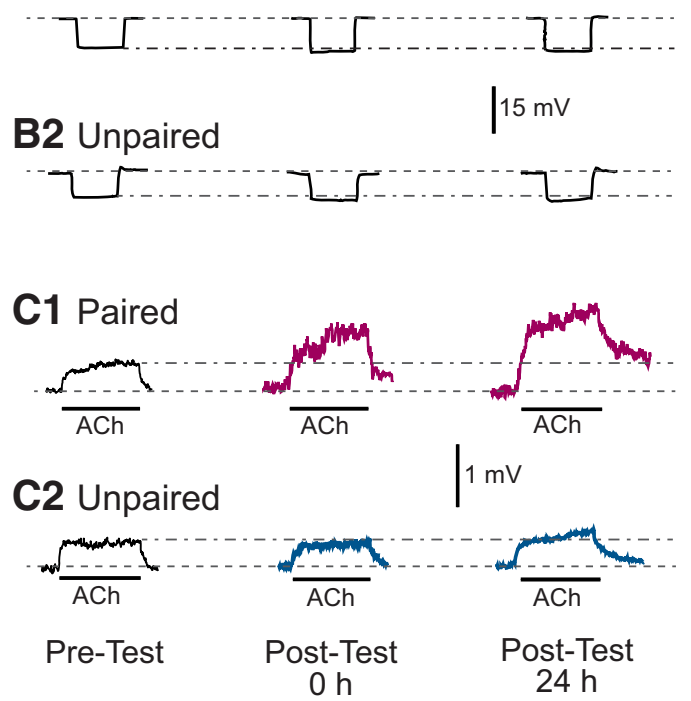

A3

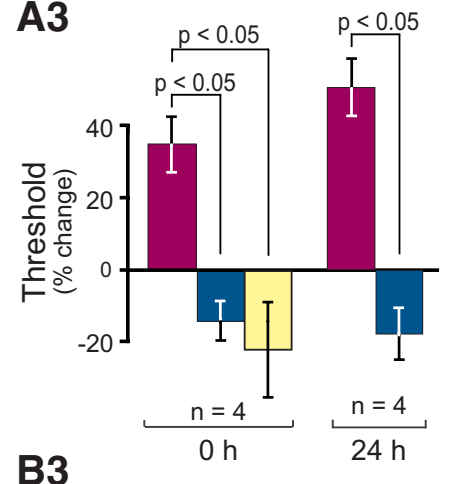

B3
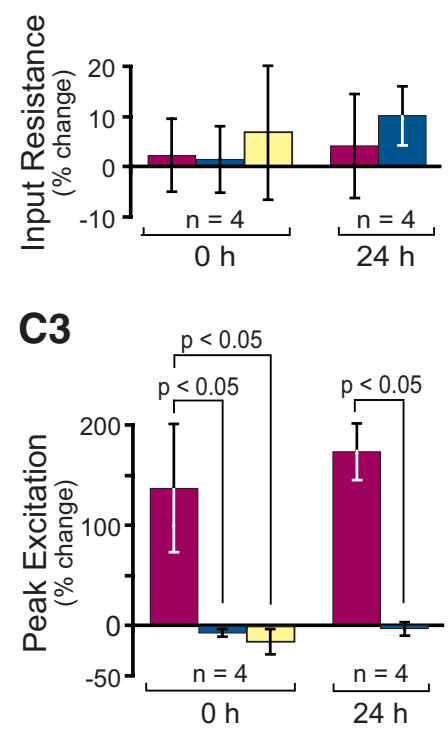

- Paired (ACh + DA)

- Unpaired (ACh + DA)

$\square$ Paired (ACh + D1 Agonist)

Figure 2. Development of the single-cell analog of classical conditioning. For each cell, the burst threshold, input resistance, and response to ACh were measured before (PreTest), immediately after (Post-Test, $0 \mathrm{~h}$ ), and $24 \mathrm{~h}$ after (Post-Test, $24 \mathrm{~h}$ ) the single-cell analog of classical conditioning. For the US, both DA and chloro-APB ( $D_{1} R$ agonist) were tested. $\boldsymbol{A 1}, \boldsymbol{A 2}$, Representative intracellular recordings from B51 illustrating the measurement of the burst threshold. In these two examples, the burst threshold increased in the paired group from $0.6 \mathrm{nA}$ initially to $0.9 \mathrm{nA}$ immediately after training and to $1.0 \mathrm{nA} 24 \mathrm{~h}$ after training $(\boldsymbol{A} \boldsymbol{1})$, whereas the threshold decreased slightly from $0.7 \mathrm{nA}$ initially to $0.6 \mathrm{nA} 24 \mathrm{~h}$ after training in the unpaired control group (A2).A3, Summary data. A significant difference was observed among the three groups (ACh paired with DA, unpaired, and ACh paired with $D_{1} R$ agonist) immediately after conditioning ( $p<0.05$, Kruskal-Wallis test). A post hoc analysis revealed a significant increase in the burst threshold in the group that received ACh paired with DA compared with either the unpaired control ( $p<0.05$, NewmanKeuls test) or the group that received ACh paired with the $D_{1}$ agonist ( $p<0.05$, Newman-Keuls test). The increase in the burst threshold was still present $24 \mathrm{~h}$ after conditioning in the ACh paired with DA group, compared with the unpaired group $(p<0.05$, Mann-Whitney test). B1, B2, Representative measurements of the input resistance of B51. B3, Summary data. No significant differences were observed between the three groups ( $A C h$ paired with $D A$, unpaired, and $A C h$ paired with $D_{1} R$ agonist) immediately after conditioning or between the two groups (ACh paired with DA, and unpaired) $24 \mathrm{~h}$ after conditioning. C1, C2, Representative intracellular recordings from B51 illustrating the responses to ACh delivery. C3, Summary data. A significant difference was observed among the three groups immediately after conditioning ( $p<0.05$, Kruskal-Wallis test). A post hoc analysis revealed a significant increase in the $A C h$ response in the group that received $A C h$ paired with DA compared with either the unpaired control $\left(p<0.05\right.$, Newman-Keuls test) or the group that received ACh paired with the $\mathrm{D}_{1}$ R agonist $(p<0.05$, Newman-Keuls test). The increase in the ACh response persisted $24 \mathrm{~h}$ after conditioning in the ACh paired with DA group, compared with the unpaired group $(p<0.05$, Mann-Whitney test).

two independent samples were made using the Mann-Whitney test. Comparisons between three independent samples were made using the Kruskal-Wallis test, and post hoc pairwise multiple comparisons were made using the nonparametric Newman-Keuls test.

\section{Results}

The first step in developing a single-cell analog of classical conditioning was the identification of the transmitters that mediate the CS and US. Previous behavioral studies used tactile stimulation of the lips (CS) paired with ingestion of food (US) (Lechner et al., 2000; Lorenzetti et al., 2006), and these stimuli were mimicked by nerve stimulation in an in vitro analog of classical conditioning consisting of the isolated cerebral and buccal ganglia (Mozzachiodi et al., 2003; Reyes et al., 2005; Lorenzetti et al., 2006). Stimulation of the AT4, which innervates the lips was used to mimic the CS, whereas stimulation of the anterior branch of the esophageal nerve (E n.2), which likely mediates food reward (Lechner et al., 2000; Brembs et al., 2002), was used to mimic the US. Two lines of evidence suggested that DA mediates the US. First, E n.2 largely consists of catecholamine-containing fibers (Kabotyanski et al., 1998). Second, a DA receptor antagonist (methylergonovine) blocked in vitro appetitive classical conditioning (Reyes et al., 2005). Thus, DA was used as a surrogate for the US. The transmitter that mediated the CS was unknown, however. AT4 stimulation produced a complex PSP in B51 with an initial inhibitory phase followed by an excitatory phase. The connection between AT4 and B51 is likely polysynaptic because a highdivalent solution blocked the PSP (data not shown). Following both in vivo and in vitro classical conditioning, the excitatory phase in B51 was enhanced, but the inhibitory phase was unchanged (Lorenzetti et al., 2006). Thus, to construct the single-cell analog of classical conditioning it was necessary to determine which transmitter mediated the excitatory phase in B51.

ACh was chosen as a candidate for the CS because ACh is a common transmitter in the feeding circuit (Church and Lloyd, 1994) and because ACh produced an excitatory response in cultured B51 (see Fig. 2C). We began by examining the effects of an ACh receptor antagonist (HEX) on AT4-elicited complex PSPs in B51 (Fig. 1). Hexamethonium was bath applied to cerebral/buccal ganglia preparations, and the AT4-elicited complex PSP was recorded in B51. The excitatory phase was selectively blocked by hexamethonium, but it recovered following washout of the drug (Fig. 1A). The peak amplitudes of the inhibitory and the excitatory phases were not significantly changed when a second AT4 stimulus was given $10 \mathrm{~min}$ after the first, showing that the responses were stable across the two stim- 
ulations (Fig. $1 B$; HEX $0 \mu \mathrm{M}$ ). The peak amplitude of the inhibitory phase was not significantly altered by hexamethonium at concentrations of either 10 or $100 \mu \mathrm{M}$ (Fig. 1B2). The excitatory phase was partially blocked by $10 \mu \mathrm{M}$ hexamethonium and was blocked to a greater extent by $100 \mu \mathrm{M}$ (Fig. 1 B1). The excitatory phase recovered following washout for each concentration. In addition, there appeared to be an increase in the frequency of IPSPs in the presence of HEX in the sample trace (Fig. 1). However, this change in the IPSP frequency was unlikely to account for the observed decrease in EPSP amplitude in HEX because the EPSP amplitude recovered to near-baseline levels following washout of HEX, where the IPSP frequency was still elevated. Because these results suggested that the excitatory phase in B51 was mediated by $\mathrm{ACh}, \mathrm{ACh}$ was chosen as a surrogate for the CS in the single-cell analog of classical conditioning.

The conditioning protocol induced a significant pairing-specific increase in the threshold for eliciting a plateau potential in B51 (Fig. 2A). The increase in threshold persisted for at least $24 \mathrm{~h}$ after training. However, there was no significant difference in the input resistance following paired training measured either by hyperpolarizing pulses (Fig. $2 B$ ) or with depolarizing pulses (paired $=-0.4 \%$, unpaired $=-3.8 \% ; p=$ 1 , Mann-Whitney test), and there was no significant difference in the holding potential following paired training (paired = $+1.2 \%$, unpaired $=+0.4 \% ; p=0.48$, Mann-Whitney test). The pairing procedure also induced an increase in the response to ACh compared with the unpaired control, and this increase persisted for $24 \mathrm{~h}$ (Fig. $2 \mathrm{C}$ ). These changes to the membrane properties and CS response of B51 were similar to what was observed following both in vivo and in vitro classical conditioning (Lorenzetti et al., 2006).

Next, the $\mathrm{D}_{1} \mathrm{R}$ agonist chloro-APB was substituted for DA. This agonist was used previously to mimic the reward signal in the single-cell analog of operant conditioning (Lorenzetti et al., 2008). However, pairing ACh with the $D_{1} R$ agonist failed to induce any significant changes in the burst threshold, input resistance, or the CS response (Fig. 2A3,B3,C3, yellow bars), suggesting that the US is mediated by either a $D_{2}$-like receptor $\left(D_{2}, D_{3}, D_{4}\right)$ or a combination of $D_{1}$-like $\left(D_{1}, D_{5}\right)$ and $D_{2}$-like receptors.

\section{Discussion}

The present study extends our analyses into the mechanistic similarities and differences between appetitive forms of classical and operant conditioning. Previously, a single-cell analog for operant conditioning was developed (Brembs et al., 2002; Lorenzetti et al., 2008) that successfully recapitulated the increase in excitability observed in B51 following both in vivo and in vitro analogs of operant conditioning (Nargeot et al., 1999b; Brembs et al., 2002; Mozzachiodi et al., 2008). This single-cell analog of operant con-
Operant Conditioning Classical Conditioning
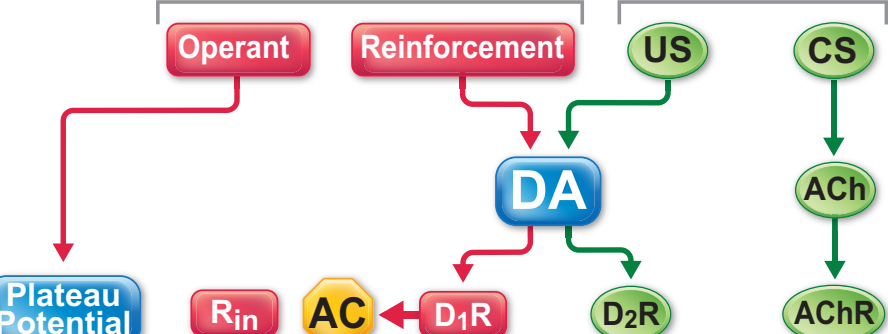

Potential

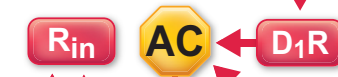

$1 R$

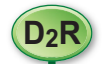

AChR

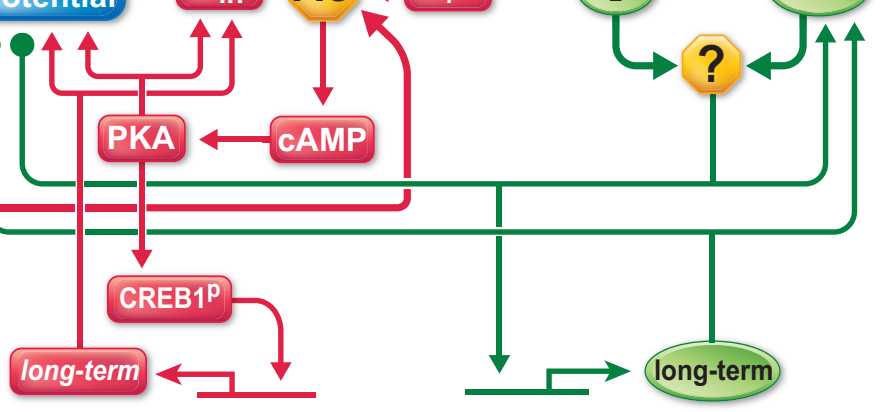

Figure 3. Putative model of the mechanisms underlying operant and classical conditioning in neuron B51. Operant conditioning: In this model, the operant is represented by a plateau potential. In turn, the plateau potential produces an accumulation of (a) o operant and classical conditioning induce long-term changes in B51. Consistent with the induction of long-term memory, the conditioning are unknown. Red components of the model are associated with operant conditioning, and green components are forcement, or the CS and US. Blue components represent sites that overlap between operant and classical conditioning. Arrows represent an enhancement or positive interaction, whereas filled circles represent an inhibition or negative interaction.

ditioning was used to elucidate the cellular and molecular mechanisms underlying operant conditioning (Lorenzetti et al., 2008). Here we report the development of a single-cell analog for classical conditioning, using ACh as the CS and DA as the US. ACh is believed to play a role in other models of classical conditioning such as eyeblink conditioning (Christian and Thompson, 2003), contextual fear learning (Raybuck and Gould, 2010), auditory conditioning (Butt et al., 2009), and olfactory learning in Drosophila (Gervasi et al., 2010). Dopamine has long been implicated as playing a role in the reward pathway (Schultz, 2002), which serves as the US in appetitive protocols. The single-cell analog described in this report successfully recapitulated the decrease in excitability and the increase in CS response in B51 that were observed following both in vivo training and in vitro analogs of classical conditioning (Lorenzetti et al., 2006). The success of the single-cell analog of classical conditioning demonstrated that the changes in B51 excitability were intrinsic to the cell. The singlecell analog of operant conditioning also induced intrinsic changes to B51 (Brembs et al., 2002). However, the operant protocol increased the excitability of B51, whereas the classical protocol decreased the excitability. Moreover, the single-cell analogs of operant and classical conditioning induced changes in B51 that persisted for at least $24 \mathrm{~h}$ (Fig. 2) (Brembs et al., 2002; Mozzachiodi et al., 2008). The ability to induce long-term changes in 
isolated B51 cells will allow analyses of long-term memory at the cellular and molecular level. For example, the single-cell analog of operant conditioning increased the levels of phosphorylated CREB1 in B51 (Lorenzetti et al., 2008). Finally, classical and operant conditioning differentially modify the intrinsic excitability of B51. Thus, the development of complementary cellular analogs with B51 for both operant and classical conditioning will facilitate a direct mechanistic comparison of the subcellular machinery that mediates these two forms of associative learning (Fig. 3). For example, a $D_{1} R$ agonist mimicked the reward signal and increased B51 excitability when used as the reinforcement in the single-cell analog of operant conditioning. However, the same $\mathrm{D}_{1} \mathrm{R}$ agonist failed to mimic the US when paired with $\mathrm{ACh}$ and failed to alter the excitability of $\mathrm{B} 51$ or the CS response in the single-cell analog of classical conditioning.

\section{References}

Baxter DA, Byrne JH (2006) Feeding behavior of Aplysia: a model system for comparing cellular mechanisms of classical and operant conditioning. Learn Mem 13:669-680.

Brembs B, Lorenzetti FD, Reyes FD, Baxter DA, Byrne JH (2002) Operant reward learning in Aplysia: neuronal correlates and mechanisms. Science 296:1706-1709.

Butt AE, Chavez CM, Flesher MM, Kinney-Hurd BL, Araujo GC, Miasnikov AA, Weinberger NM (2009) Association learning-dependent increases in acetylcholine release in the rat auditory cortex during auditory classical conditioning. Neurobiol Learn Mem 92:400-409.

Christian KM, Thompson RF (2003) Neural substrates of eyeblink conditioning: acquisition and retention. Learn Mem 10:427-455.

Church PJ, Lloyd PE (1994) Activity of multiple identified motor neurons recorded intracellularly during evoked feedinglike motor programs in Aplysia. J Neurophysiol 72:1794-1809.

Gervasi N, Tchénio P, Preat T (2010) PKA dynamics in a Drosophila learning center: coincidence detection by rutabaga adenylyl cyclase and spatial regulation by dunce phosphodiesterase. Neuron 65:516-529.

Jing J, Cropper EC, Hurwitz I, Weiss KR (2004) The construction of movement with behavior-specific and behavior-independent modules. J Neurosci 24:6315-6325.

Kabotyanski EA, Baxter DA, Byrne JH (1998) Identification and characterization of catecholaminergic neuron B65, which initiates and modifies patterned activity in the buccal ganglia of Aplysia. J Neurophysiol 79:605-621.

Kabotyanski EA, Baxter DA, Cushman SJ, Byrne JH (2000) Modulation of fictive feeding by dopamine and serotonin in Aplysia. J Neurophysiol $83: 374-392$
Lechner HA, Baxter DA, Byrne JH (2000) Classical conditioning of feeding in Aplysia: I. Behavioral analysis. J Neurosci 20:3369-3376.

Lorenzetti FD, Mozzachiodi R, Baxter DA, Byrne JH (2006) Classical and operant conditioning differentially modify the intrinsic properties of an identified neuron. Nat Neurosci 9:17-19.

Lorenzetti FD, Baxter DA, Byrne JH (2008) Molecular mechanisms underlying a cellular analog of operant reward learning. Neuron 59:815-828.

Mozzachiodi R, Lechner HA, Baxter DA, Byrne JH (2003) In vitro analog of classical conditioning of feeding behavior in Aplysia. Learn Mem 10:478-494

Mozzachiodi R, Lorenzetti FD, Baxter DA, Byrne JH (2008) Changes in neuronal excitability serve as a mechanism of long-term memory for operant conditioning. Nat Neurosci 11:1146-1148.

Nargeot R, Simmers J (2011) Neural mechanisms of operant conditioning and learning-induced behavioral plasticity in Aplysia. Cell Mol Life Sci 68:803-816.

Nargeot R, Baxter DA, Byrne JH (1997) Contingent-dependent enhancement of rhythmic motor patterns: an in vitro analog of operant conditioning. J Neurosci 17:8093-8105.

Nargeot R, Baxter DA, Byrne JH (1999a) In vitro analog of operant conditioning in Aplysia. I. Contingent reinforcement modifies the functional dynamics of an identified neuron. J Neurosci 19:2247-2260.

Nargeot R, Baxter DA, Byrne JH (1999b) In vitro analog of operant conditioning in Aplysia. II. Modifications of the functional dynamics of an identified neuron contribute to motor pattern selection. J Neurosci 19:2261-2272.

Nargeot R, Baxter DA, Patterson GW, Byrne JH (1999c) Dopaminergic synapses mediate neuronal changes in an analogue of operant conditioning. J Neurophysiol 81:1983-1987.

Nargeot R, Petrissans C, Simmers J (2007) Behavioral and in vitro correlated of compulsive-like food seeding induced by operant conditioning in Aplysia. J Neurosci 27:8059-8070.

Raybuck JD, Gould TJ (2010) The role of nicotinic acetylcholine receptors in the medial prefrontal cortex and hippocampus in trace fear conditioning. Neurobiol Learn Mem 94:353-363.

Rescorla RA, Solomon RL (1967) Two-process learning theory: relationships between Pavlovian conditioning and instrumental learning. Psychol Rev 74:151-182.

Reyes FD, Mozzachiodi R, Baxter DA, Byrne JH (2005) Reinforcement in an in vitro analog of appetitive classical conditioning of feeding behavior in Aplysia: blockade by a dopamine antagonist. Learn Mem 12:216-220.

Schultz W (2002) Getting formal with dopamine and reward. Neuron 36:241-263

Shetreat-Klein AN, Cropper EC (2004) Afferent-induced changes in rhythmic motor programs in the feeding circuitry of Aplysia. J Neurophysiol 92:2312-2322.

Siegel S (1956) Nonparametric statistics for the biological sciences. New York: McGraw-Hill. 